\title{
Association Between Radiologic Incidental Findings and Resource Utilization in Patients Admitted With Chest Pain in an Urban Medical Center
}

\author{
Venkat P. Gundareddy, MD, MPH${ }^{1 *}$ and Nisa M. Maruthur, MD, MHS ${ }^{1-3}$, Abednego Chibungu, MD¹, Preetam Bollampally, MD4, \\ Regina Landis, $\mathrm{MS}^{1}$, Shaker M. Eid, MD, $\mathrm{MBA}^{1}$
}

\begin{abstract}
${ }^{1}$ Department of Medicine, Johns Hopkins University School of Medicine, Baltimore MD; '2Department of Epidemiology, Johns Hopkins University Bloomberg School of Public Health, Baltimore MD; 'Welch Center for Prevention, Epidemiology, \& Clinical Research, Johns Hopkins University, Baltimore MD; ${ }^{4}$ Department of Medicine, Saint Vincent Hospital, Worcester, MA.
\end{abstract}

\begin{abstract}
BACKGROUND: Increasing use of testing among hospitalized patients has resulted in an increase in radiologic incidental findings (IFs), which challenge the provision of high-value care in the hospital setting.
\end{abstract}

OBJECTIVE: To understand impact of radiologic incidental findings on resource utilization in patients hospitalized with chest pain.

DESIGN: Retrospective observational cross sectional study. SETTING: Academic medical center.

PARTICIPANTS: Adult patients hospitalized with principal diagnosis of chest pain.

MEASUREMENTS: Demographic, imaging, and length of stay (LOS) data were abstracted from the medical charts. We used multiple logistic regression to evaluate factors associated with radiologic IFs and negative binomial regression to evaluate the association between radiologic IFs and LOS.
RESULTS: 1811 consecutive admissions with chest pain were analyzed retrospectively over a period of 24 months; 376 patients were included in the study after exclusion criteria were applied and readmissions removed. Of these, 197 patients (52\%) had 364 new radiologic IFs on imaging; most IFs were of minor $(50 \%)$ or moderate clinical significance $(42 \%)$, with only $7 \%$ of major significance. Odds of finding radiologic IFs increased with age (adjusted odds ratio, 1.04; $95 \%$ confidence interval [Cl], 1.01-1.06) and was associated with a $26 \%$ increase in LOS (adjusted incidence rate ratio, 1.26; $95 \% \mathrm{Cl}, 1.07-1.49$ ).

CONCLUSION: Radiologic IFs were very common among patients hospitalized with chest pain of suspected cardiac origin and independently associated with an increase in the LOS. Interventions to address radiologic IFs may reduce LOS and, thereby, support high-value care. Journal of Hospital Medicine 2017;12:323-328. (C) 2017 Society of Hospital Medicine
Diagnostic imaging is an integral part of patient evaluation in acute care settings. The use of imaging for presenting complaints of chest pain, abdominal pain, and injuries has increased in emergency departments across the United States without an increase in detection of acute pathologic conditions. ${ }^{1,2}$ An unintended consequence of this increase in diagnostic imaging is the discovery of incidental findings (IFs).

Incidental findings are unexpected findings (eg, nodules) noted on diagnostic imaging that are not related to the presenting complaint. ${ }^{3}$ The increasing use of diagnostic imaging and increased sensitivity of these tests have led to a higher burden of radiologic IFs. ${ }^{4}$ In a tertiary level hospital, Lumbreras et al..$^{5}$ found that the overall incidence of IFs for all radiologic imaging for inpatients and outpatients was $15 \%$, while Orme et $\mathrm{al}^{6}{ }^{6}$ found that the incidence in imaging research was $39.8 \%$. The existing evidence base suggests that

\footnotetext{
*Address for correspondence and reprint requests: Venkat Pradeep Gundareddy, MD, MPH; Johns Hopkins University School of Medicine; Division of Hospital Medicine, Johns Hopkins Bayview Medical Center, 5200 Eastern Ave, MFL West 6th Floor, Baltimore, MD 21224; Telephone: 410-550-5018; Fax: 410-550-2972; E-mail: vgundar1@jhmi.edu

Additional Supporting Information may be found in the online version of this article. Received: July 12, 2016; Revised: November 5, 2016; Accepted: November 13, 2016
}

2017 Society of Hospital Medicine DOI 10.12788/jhm.2722 the identification of radiologic IFs has financial, ${ }^{5,7}$ clinical, ${ }^{6}$ ethical, and legal implications. ${ }^{8}$ Also, IFs increase workload for healthcare professionals, including that related to follow-up and surveillance. ${ }^{9}$

In the field of radiology, the burden of radiologic IFs is a well-accepted fact and various white papers have been published by the American College of Radiology on how to address them. ${ }^{4,7}$ Hospitalized patients are a population that undergoes a substantial number of diagnostic tests. In the era of accountable care organization ${ }^{10}$ with an emphasis on population health and high-value care, radiologic IFs pose a particular challenge to healthcare providers.

Chest pain is one of the most common reasons for emergency department visits in the United States. ${ }^{11}$ In this study, we report on radiologic IFs and factors associated with these among patients hospitalized for chest pain of suspected cardiac origin, and we evaluate the hypothesis that radiologic IFs are associated with an increase in LOS in this population.

\section{METHODS}

We conducted a secondary analysis of data from the Chest Pain and Cocaine Study (CPAC). The CPAC study is a cross sectional study of all patients hospitalized with chest pain to our urban academic medical center. Medical records were reviewed to generate a database of all such patients during 


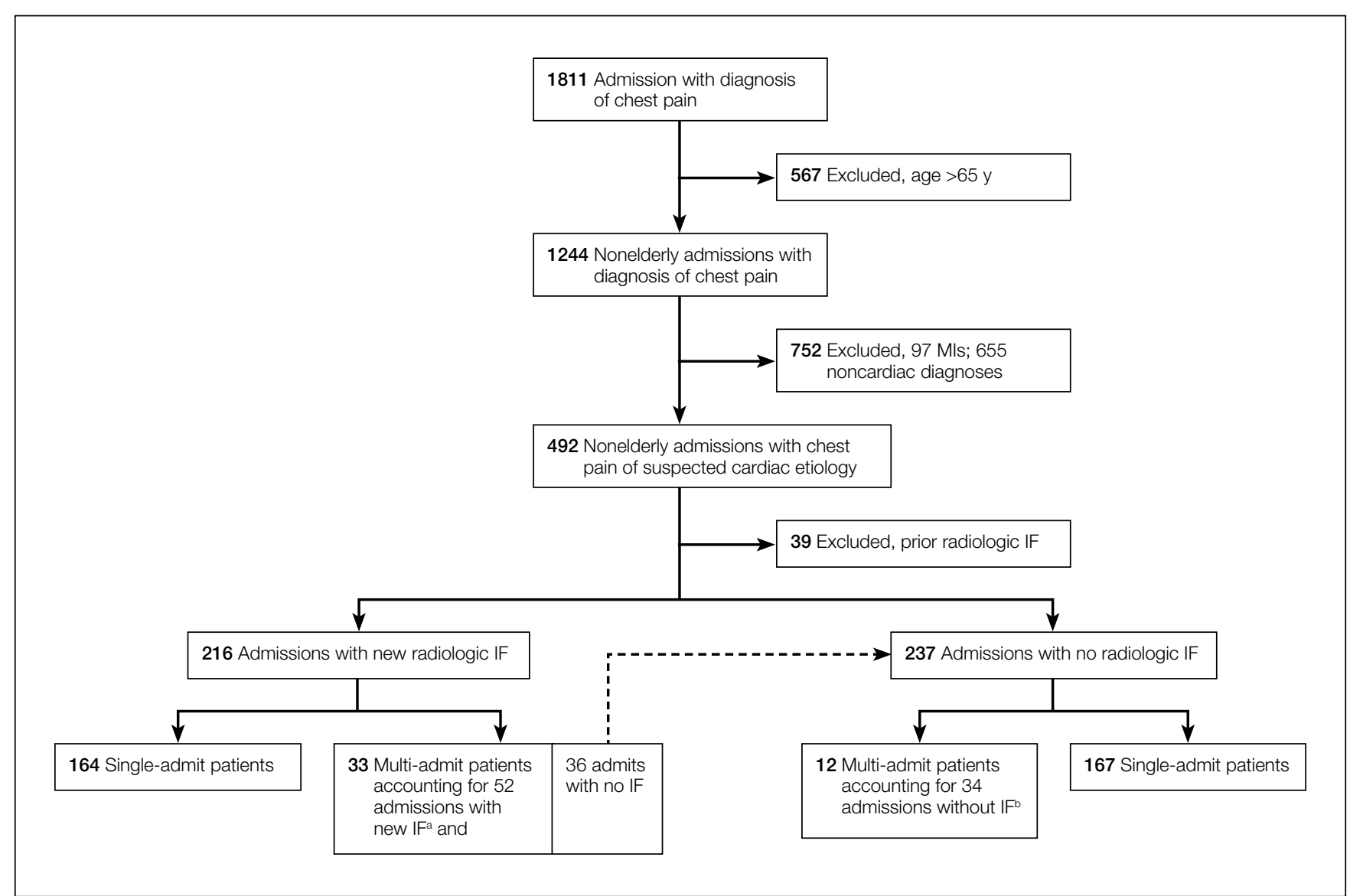

FIG. Flow chart of patient selection.

apatients with multiple admissions and at least 1 admission having radiologic IFs. Only the first admission with new radiologic IFs was included.

'Patients with multiple admissions and no radiologic IFs. Only the first admission was included.

NOTE: Abbreviations: IF, incidental findings; Ml, myocardial infarction.

the study period. The main focus of CPAC was to look at healthcare disparities and resource utilization in patients with or without a concomitant diagnosis of cocaine use. ${ }^{12}$

\section{Study Population}

The Figure shows the selection of the study sample for this analysis. The $\mathrm{CPaC}$ Study identified 1811 consecutive admissions for chest pain/angina pectoris (based on admitting diagnosis ICD-9-CM codes: 411.x; 413.x, 414.x; and 786.5x) over 24 months. Per the $\mathrm{CPaC}$ Study protocol, patients older than 65 years were excluded ( $\mathrm{n}=567$ admissions). After chart review, all admissions diagnosed with acute myocardial infarction $(n=97)$ or noncardiac chest pain $(n=655)$ were excluded. For this analysis, we excluded 39 additional admissions of patients who had known prior radiologic IFs, leading to a sample size of 453 admissions. Three hundred and seventy six patients had accounted for 453 admissions during the study period, and we included 1 of these admissions in the analysis using the following process: If a patient had a radiologic IF on any admission during the study period, that patient was included in the "IF" group for the analysis, and data from the first admission with an IF were used for the analysis. If a patient had no radiologic IFs on any admission during the study period, that patient was included in the "no IF" group, and the data from the first admission in the database were used for analysis.

\section{Measurements}

Data collection was completed retrospectively by medical record review using a standardized $\mathrm{CPaC}$ Study protocol. The database was created and maintained using REDCap (Research Electronic Data Capture; Vanderbilt University, Knoxville, Tennessee) electronic data capture tool hosted at Johns Hopkins University. ${ }^{13}$ All data were manually abstracted into REDCap from electronic medical records. All missing values and inconsistent data were reviewed by multiple physicians to ensure data integrity.

We defined all diagnostic (noninterventional; nonlaboratory) testing done during a patient's hospitalization as "diagnostic" tests, except cardiac stress testing and echocardiogram. We defined diagnostic tests as "primary" tests if they were done in response to patients' presenting complaint. We defined diagnostic tests as "secondary" tests if they were done by providers due to IFs. Cardiac computed tomography was included in diagnostic tests. Cardiac testing (echocardiogram, cardiac stress testing, cardiac catheter- 
ization and pacemaker placement) was considered separate from the "diagnostic tests" since these were focused cardiac imaging that are interventional in nature with low yield on extra-cardiac radiologic IFs.

Incidental findings were defined as any unexpected findings on diagnostic imaging unrelated to the reason for admission, and were classified based on organ systems and their clinical significance as major, moderate, or minor using a classification previously published by Lumbreras et al. ${ }^{14}$ All radiologic IFs data underwent sequential dual review by investigators for accuracy of documentation. Individuals with multiple radiologic IFs belonging to more than one category of clinical significance were categorized with the IFs group of highest clinical significance. Ten percent of the patients with no IFs were reviewed again, and no errors found.

Demographic variables at the time of admission included age, sex, race, level of education, employment status, insurance status, body mass index (BMI), and smoking status. Comorbid conditions at the time of admission consisted of the following: hypertension, diabetes mellitus, chronic kidney disease (CKD), chronic obstructive pulmonary disease (COPD), history of myocardial infarction, cerebrovascular accident (CVA), congestive heart failure (CHF), drug use and malignancy or history of it. Initial laboratory values were extracted from electronic medical records and included hemoglobin, creatinine, blood urea nitrogen (BUN), aspartate transaminase, alanine transaminase, and alkaline phosphatase. We calculated the estimated glomerular filtration rate (eGFR) using the MDRD (Modification of Diet in Renal Disease) equation..$^{15}$ Admission and discharge information as well as whether the patient had a primary care provider, were obtained from medical records. The length of hospital stay was calculated by subtracting date of admission from date of discharge.

\section{Statistical Analysis}

We conducted 2 main analyses: 1) a descriptive analysis of the association between patient characteristics (independent variables) and identification of IFs during admission (primary outcome) and 2) an analysis of the association between identification of incidental findings during admission (independent variable) and LOS (primary outcome).

For the descriptive analysis of radiologic IFs, we compared the characteristics of patients with and without radiologic IFs during admission using a t-test (for normally distributed continuous variables) or Mann-Whitney test (for nonnormally distributed continuous variables) and a chi-square or Fisher exact test for categorical variables based on the number of observations. We included variables significantly associated with the occurrence of radiologic IFs $(P<0.05)$ in a multiple logistic regression model to identify characteristics independently associated with presence of radiologic IFs.

Length of stay was right-skewed even after natural logarithm transformation and, therefore, we used negative binomial regression for the analysis of the association between the identification of radiologic IFs during admission and LOS.
We included potential confounding variables in the multiple negative binomial regression model based on plausibility of confounding and association with both the exposure (identification of radiologic IFs during admission) and outcome (LOS) at a level of $P<0.3$. Age, education level, history of drug use, history of CHF, history of CKD, lower eGFR, higher serum creatinine/BUN, hemoglobin, occurrence of cardiac catheterization, stress testing, and multiple admissions during the study period were identified as confounders. For correlated variables (eg, hemoglobin and hematocrit), the variable with the strongest statistical association (lowest $P$ value) was included in the model. In sensitivity analysis, we dropped patients with extreme LOS (longer than 10 days). All analyses were performed using STATA 13 (Stata Statistical Software: Release 13; StataCorp., College Station, Texas).

\section{RESULTS}

Table 1 shows the characteristics of the 376 patients included in this study. Overall mean age was 50.5 years, $40 \%$ were females, $62 \%$ were Caucasian, $66 \%$ were unemployed, $84 \%$ identified a primary care provider upon admission, and $68 \%$ were cared for by a hospitalist. Overall median LOS was 2 days (interquartile range $[\mathrm{IQR}]=2$ ). Of the 376 patients in the study, 197 (52\%) had new radiologic IFs. Comparing the patients with radiologic IFs and no IFs, it was evident that more radiological tests were performed in the IF group (2.2 tests per patient) in comparison with the no IF group (1.26 tests per patient). Looking at patient characteristics, patients with radiologic IFs were older ( 52 years vs. 48.8 years; $P<0.001$ ), reported a lower education level and lower hemoglobin levels on admission $(12.0 \mathrm{gm} / \mathrm{dL}$ vs. $13.4 \mathrm{gm} / \mathrm{dL}$; $P=0.029$ ), but were more likely to be unemployed (72\% vs. $59 \% ; P=0.009)$, have COPD ( $19 \%$ vs. $10 \% ; P=0.007)$, and a history of malignancy ( $7 \%$ vs. $2 \%, P=0.04)$. In addition, patients in the radiologic IF group had lower rates of cardiac catheterization ( $18 \%$ vs. $28 \% ; P=0.02)$, were more likely to be readmitted more than once during the study period $(17 \%$ vs. $7 \% ; P=0.02)$ and be discharged by hospitalists $(75 \%$ vs. $60 \% ; P=0.003$; Supplemental Table 1).

Overall, 658 diagnostic tests were performed in the study population; of these, $268(40.7 \%)$ tests revealed 364 new radiologic IFs (Supplement Table 2). Of these radiologic IFs, $27(7.4 \%)$ were of major clinical significance, 154 (42\%) were of moderate clinical significance, and 183 (50\%) were of minor clinical significance (Supplement Table 3). Computed tomography (CT) scans yielded more IFs compared to any other imaging modalities. Of the radiologic IFs of major clinical significance, 3 malignant/premalignant lesions were found. While pulmonary nodules were the most common moderate clinically significant findings, atelectasis and spinal degenerative changes were the most common radiologic IFs of minor clinical significance (Supplement Table 4).

Results of the logistic regression models testing the association between patient characteristics and radiologic IFs are displayed in Table 2. Only age and repeat admissions 


\begin{tabular}{|c|c|}
\hline Patient Characteristics & $\begin{array}{l}\text { Total Study Population, } \\
\qquad \mathrm{N}=376(\%)\end{array}$ \\
\hline Age, y & $50.5 \pm 9.3$ \\
\hline Female & $151(40)$ \\
\hline \multicolumn{2}{|l|}{ Race } \\
\hline White & $234(62)$ \\
\hline Black & $123(33)$ \\
\hline Other (Asian, Hispanic) & $19(5)$ \\
\hline Unemployed & $248(66)$ \\
\hline \multicolumn{2}{|l|}{ Insurance } \\
\hline Medicare & $79(21)$ \\
\hline Medicaid & $73(19)$ \\
\hline Private insurance & $187(50)$ \\
\hline Self-pay & $37(10)$ \\
\hline \multicolumn{2}{|l|}{ Education level } \\
\hline Not completed high school & $76(20)$ \\
\hline Completed high school & $222(59)$ \\
\hline Completed college & $78(21)$ \\
\hline $\mathrm{BMl}, \mathrm{kg} / \mathrm{m}^{2}$ & $31.5 \pm 8.1$ \\
\hline Hemoglobin level, gm/dL & $13.2 \pm 1.9$ \\
\hline eGFR on admission, $\mathrm{mL} / \mathrm{min} / 1.73 \mathrm{~m}^{2}$ & $81.8 \pm 35.4$ \\
\hline ALT on admission, units/liter & $40.8 \pm 40$ \\
\hline \multicolumn{2}{|l|}{ Tobacco use } \\
\hline Never smoker & $133(36)$ \\
\hline Past smoker & $69(18)$ \\
\hline Active smoker & $174(46)$ \\
\hline Hypertension & $262(70)$ \\
\hline Diabetes mellitus & $136(36)$ \\
\hline$\overline{C K D}$ & $66(18)$ \\
\hline Prior Ml & $82(22)$ \\
\hline COPD & $55(15)$ \\
\hline $\mathrm{CHF}$ & $50(13)$ \\
\hline CVA & $46(12)$ \\
\hline Prior diagnosis of malignancy & $17(5)$ \\
\hline Drug use & $76(20)$ \\
\hline PCP present on admission & $317(84)$ \\
\hline Cardiac catheterization during current admission & $85(23)$ \\
\hline Stress test during current admission & $163(43)$ \\
\hline Echocardiogram during current admission & $89(24)$ \\
\hline \multicolumn{2}{|l|}{ Discharging provider } \\
\hline House staff & $112(30)$ \\
\hline NP/PA & $9(2)$ \\
\hline Hospitalist & $255(68)$ \\
\hline $\operatorname{LOS}(d)^{\mathrm{a}}$ & $2(2)$ \\
\hline \multicolumn{2}{|l|}{ Discharge location } \\
\hline Home & $345(92)$ \\
\hline Other (nursing home, rehabilitation facility, or shelter) & $31(8)$ \\
\hline Patients with multiple admissions during study period & $45(12)$ \\
\hline \multicolumn{2}{|c|}{$\begin{array}{l}\text { Abbreviations: ALT, alanine transaminase; BMI, body mass index; CHF, congestive heart failure; CVA, cerebro- } \\
\text { vascular accident; COPD, chronic obstructive pulmonary disease; eGFR, estimated glomerular filtration rate; IF, } \\
\text { incidental findings; LOS, length of stay; MI, myocardial infarction; NP, nurse practitioner; PA, physician assistant; } \\
\text { PCP, primary care provider. }\end{array}$} \\
\hline
\end{tabular}

remained significantly associated with radiologic IFs in the fully adjusted model (adjusted odds ratio [OR], 1.04; 95\% confidence interval [CI], 1.01-1.06 and 2.68; $95 \%$ CI, 1.60 4.44, respectively).

Median LOS was 2 days $(\mathrm{IQR}=1)$ for patients with no IFs and 2 days $(I Q R=2)$ for patient with radiologic IFs $(P$ $=0.08)$. Unadjusted negative binomial regression analysis revealed that identification of any radiologic IFs during admission (vs. none) was associated with an increased LOS by $24 \%$ (unadjusted IRR, 1.24; $95 \%$ CI, 1.06-1.45). After adjustment for confounders, identification of any radiologic IFs during admission remained significantly associated with a longer LOS (adjusted IRR, 1.26; 95\% CI, 1.07-1.49). Results remained significant on a sensitivity analysis excluding admissions lasting longer than 10 days (adjusted IRR, 1.21; 95\% CI, 1.03-1.42; Supplement Table 5).

Incidental findings of minor and moderate clinical significance were associated with increase in LOS on multiple negative binomial regression (adjusted IRR, 1.27; 95\% CI, 1.03-1.57 and 1.24; 95\% CI, 1.02-1.52, respectively; Table $3)$; however, upon dropping length of hospitalization outliers, only radiologic IFs with major clinical significance were associated with increase in length of hospitalization (adjusted IRR, 1.39; 95\% CI, 1.04-1.87; Table 3).

Supplemental chart review revealed that 26 patients accounted for the 27 radiologic IFs of major clinical significance. This group had 54\% women, median LOS remained 2 days (IQR 2) and, on average, had about 3 diagnostic tests performed per patient. Cardiac testing was performed less on these patients compared to others (Supplement Table S6). Review also revealed that, of the 26 patients, 2 had abnormal labs, 2 had drug abuse/psychiatric issues, and another 2 had radiologic IFs that warranted further consultations, imaging, and longer LOS.

\section{DISCUSSION}

Radiologic IFs in patients admitted with chest pain of suspected cardiac origin are a common occurrence as shown in our study. Similar to prior studies, $41 \%$ of all radiologic tests done in our study population revealed IFs. ${ }^{6}$ The majority of the IFs were of minor to moderate clinical significance and, as reported in the literature, were more common with older age and CT imaging. ${ }^{14,16}$ In addition, an IF diagnosed during admission for chest pain was associated with a $26 \%$ increase in length of hospital stay.

To our knowledge, we present the first study on the impact of identification of radiologic IFs in hospitalized patients on length of hospital stay and specifically in patients hospitalized with chest pain of suspected cardiac origin. Trends over the past decade have shown a decrease in LOS and hospitalizations but with an increase in health resource utilization. ${ }^{17,18}$ Association of radiologic IFs with increase in LOS is significant as this potentially increases hospital-acquired conditions such as infections and resource utilization leading to increase in costs of hospitalizations. ${ }^{19}$ This in return is a concern for patient safety. 
TABLE 2. Logistic Regression Analysis of Factors Associated with IFs

\begin{tabular}{|c|c|c|c|c|c|c|}
\hline \multirow[b]{3}{*}{ Variable } & \multicolumn{3}{|c|}{ Univariate Regression } & \multicolumn{3}{|c|}{ Multivariate Regression } \\
\hline & & & & Adjusted & & \\
\hline & Unadjusted OR & $\mathrm{Cl}$ & $P$ value & OR & $\mathrm{Cl}$ & $P$ value \\
\hline Unemployment & 0.56 & $0.36-0.86$ & 0.009 & 0.88 & $0.54-1.44$ & 0.626 \\
\hline \multicolumn{7}{|l|}{ Education: } \\
\hline Not completed high school & (1.0) ref & & & (1.0) ref & & \\
\hline Multiple admissions during study period & 2.68 & $1.64-4.35$ & $<0.001$ & 2.68 & $1.60-4.44$ & $<0.001$ \\
\hline$\overline{\mathrm{COPD}}$ & 2.28 & $1.23-4.20$ & 0.08 & 1.4 & $0.71-2.76$ & 0.33 \\
\hline Prior diagnosis of malignancy & 3.09 & $0.99-9.66$ & 0.05 & 1.66 & $0.46-6.07$ & 0.435 \\
\hline Cardiac catheterization & 0.56 & $0.34-0.91$ & 0.019 & 0.65 & 0.36-1.16 & 0.146 \\
\hline Hospitalist & 1.91 & $1.22-3.00$ & 0.005 & 1.62 & $0.97-2.72$ & 0.065 \\
\hline
\end{tabular}

The positive association between LOS and radiologic IFs, interestingly, continued to exist despite sensitivity analysis. Incidental findings of major clinical significance were associated with longer LOS in the sensitivity analysis. Supplemental chart review of patients with major clinical findings suggested more extra-cardiac workup compared to patients with minor/moderate radiologic IFs. This could indicate that the presence of clinically significant radiologic IFs could have led to further inpatient work-up and consultations. The downstream healthcare expenditure associated with workup of IFs in individual radiologic tests is well established..$^{20}$ In case of cardiac CT, Goehler et al. ${ }^{21}$ found that the healthcare expenditure was high following incidentally detected pulmonary nodules with an overall small reduction in lung cancer mortality. Incidental findings also increase the burden of reporting and concern for medico-legal issues for providers. ${ }^{4}$ These concerns are likely valid for hospitalized patients as well.

The socioeconomic trends in the study population were consistent with data from the Bureau of Labor Statistics in that low education is associated with higher unemployment. ${ }^{22}$ Although, overall, gender, race and insurance mix were similar in both groups, we did see trends of socioeconomic differences in the patients with radiologic IFs of major clinical significance that might not have been statistically significant owing to the small sample size. Despite the population being relatively of younger age (given our cut off age was 65 years) there was still a positive association with age and presence of radiologic IFs. The higher number of patients with COPD or history of malignancy in the radiologic IF group suggests that an association with IFs could exist for these disease cohorts; however, after adjustment for multiple covariates, such an association did not transpire. Interestingly, patients with no radiologic IFs underwent cardiac catheterization or stress testing more often than patients
TABLE 3. Multivariate Negative Binomial Regression of LOS by IF Clinical Significance

\begin{tabular}{lccc}
\hline Clinical Significance & $\mathrm{IRR}^{\mathrm{a}}(\mathrm{LOS})$ & $\mathrm{Cl}$ & $P$ value \\
\hline No IF & $(1.0)$ ref & & \\
Minor & 1.27 & $1.03-1.57$ & 0.023 \\
Moderate & 1.24 & $1.02-1.52$ & 0.031 \\
Major & 1.33 & $0.98-1.82$ & 0.071 \\
\hline Drop LOS $>10 \mathrm{~d}$ & & & \\
No IF & $(1.0)$ ref & & \\
Minor & 1.16 & $0.94-1.42$ & 0.15 \\
Moderate & 1.20 & $0.99-1.46$ & 0.057 \\
Major & 1.39 & $1.04-1.87$ & 0.027 \\
\hline
\end{tabular}

adjusted for age, history of drug abuse, history of congestive heart failure, history of cerebrovascular accident/ transient ischemic attack, body mass index, multiple admissions during study period, and cardiac catheterization/ cardiac stress test during admission, creatinine, education level, and hemoglobin.

NOTE: Abbreviations: $\mathrm{Cl}$, confidence interval; IF, incidental finding; IRR, incident rate ratio; LOS, length of stay; ref, referent.

with discovered IFs. This speaks of 2 possibilities; first, that both tests probably do not yield many extra-cardiac IFs, or, secondly, that these patients did not require further workup. More patients in the IF group had more than 1 admission during the study period, and this was associated with increased odds of detecting radiologic IFs. We hypothesize that this might have occurred because of the diagnostic dilemma in these patients who have multiple admissions for the same reason leading to wider array of diagnostic workup. Indeed, we did not note upon chart review alternative diagnoses in these patients but only more IFs. There are several study limitations to consider. First, the fact that this is a single center study sets limitations to interpretation and generalizability of the data. Second, we cannot exclude the possibility of residual confounding. Third, the small number of patients included in this study precludes definitive identification of more factors potentially associated with IFs. However, this 
study sheds light on a yet unidentified problem within the realm of inpatient management especially for the internists and hospitalists. We tried to limit bias to the extent possible by including only 1 presenting complaint and age-restricting the population.

\section{CONCLUSION}

Incidental findings are both clinical and financial challenges to the medical field. This study attempted to shed light on impact of radiologic IFs on care and resource utilization in patients admitted with chest pain of suspected cardiac origin. The positive association between radiologic IFs and length of hospital stay implies that the presence of IFs is associated with increase in LOS and indirectly a likely increase in overall healthcare expenditure. Given the high incidence of radiologic IFs, assuming that these will be present on radiologic tests, should be more a norm than an exception. Providers should know that radiologic testing, especially $\mathrm{CT}$, is associated with detection of IFs. ${ }^{16}$ By avoiding inappropriate ordering of imaging, the issue of IFs could be mitigated.

While radiologists have recommendations about necessary follow-up for some IFs, ${ }^{7}$ no clear follow-up guidelines exist for most IFs arising in hospitalized patients. Further prospective and cost analysis studies are needed to assess the overall impact of IFs on other hospitalized patient populations and on the healthcare system in general.

Disclosure: The authors report no conflicts of interest.

\section{References}

1. Korley FK, Pham JC, Kirsch TD. Use of advanced radiology during visits to US emergency departments for injury-related conditions, 1998-2007. JAMA. 2010;304(13):1465-1471.

2. Pines JM. Trends in the rates of radiography use and important diagnoses in emergency department patients with abdominal pain. Med Care. 2009;47(7):782-786.

3. McGraw-Hill Concise Dictionary of Modern Medicine. Incidentalomas. http:// medical-dictionary.thefreedictionary.com/Incidental+findings. Updated 2002. Accessed April 13, 2017.

4. Berland LL, Silverman SG, Gore RM, et al. Managing incidental findings on ab dominal CT: White paper of the ACR incidental findings committee. J Am Coll Radiol. 2010;7(10):754-773.

5. Lumbreras B, González-Alvárez I, Lorente MF, Calbo J, Aranaz J, Hernán-
dez-Aguado I. Unexpected findings at imaging: Predicting frequency in various types of studies. Eur J Radiol. 2010;74(1):269-274.

6. Orme NM, Fletcher JG, Siddiki HA, et al. Incidental findings in imaging research: Evaluating incidence, benefit, and burden. Arch Intern Med. 2010;170(17):1525-1532.

7. Berland LL. Overview of white papers of the ACR incidental findings committee II on adnexal, vascular, splenic, nodal, gallbladder, and biliary findings. J Am Coll Radiol. 2013;10(9):672-674.

8. Booth TC, Jackson A, Wardlaw JM, Taylor SA, Waldman AD. Incidental findings found in "healthy" volunteers during imaging performed for research: Current legal and ethical implications. Br J Radiol. 2010;83(990):456-465.

9. Kelly ME, Heeney A, Redmond CE, et al. Incidental findings detected on emergency abdominal CT scans: A 1-year review. Abdom Imaging. 2015;40(6):1853-1857.

10. Centers for Medicare and Medicaid Services. Accountable care organizations (ACO). https://www.cms.gov/Medicare/Medicare-Fee-for-Service-Payment/ ACO/index.html?redirect=/aco. Baltimore, Maryland. Updated 01/06/2015.

11. Weiss AJ (Truven Health Analytics), Wier LM (Truven Health Analytics), Stocks C (AHRQ), Blanchard J (RAND). Overview of Emergency Department Visits in the United States, 2011. HCUP Statistical Brief \#174. June 2014. Agency for Healthcare Research and Quality, Rockville, MD. http://www.hcup-us.ahrq. gov/reports/statbriefs/sb174-Emergency-Department-Visits-Overview.pdf.

12. Chibungu A, Gundareddy V, Wright SM, Nwabuo C, Bollampally P, Landis R, Eid SM. Management of cocaine-induced myocardial infarction: 4-year experience at an urban medical center. South Med J. 2016;109(3):185-190.

13. Harris PA, Taylor R, Thielke R, Payne J, Gonzalez N, Conde JG. Research electronic data capture (REDCap)--a metadata-driven methodology and workflow process for providing translational research informatics support. J Biomed Inform. 2009; $42(2): 377-381$

14. Lumbreras B, Donat L, Hernández-Aguado I. Incidental findings in imaging diagnostic tests: a systematic review. Br J Radiol. 2010;83(988):276-289.

15. Fontela PC, Winkelmann ER, Ott JN, Uggeri DP. Estimated glomerular filtration rate in patients with type 2 diabetes mellitus. Rev Assoc Méd Bras (1992). 2014;60(6):531-537.

16. Samim M, Goss S, Luty S, Weinreb J, Moore C. Incidental findings on CT for suspected renal colic in emergency department patients: prevalence and types in 5,383 consecutive examinations. J Am Coll Radiol. 2015;12(1):63-69.

17. Avalere Health for the American Health Association. TrendWatch ChartBook 2014; trends affecting hospitals and health systems. 2014. http://www.aha.org/research/reports/tw/chartbook/2014/14chartbook.pdf.

18. Weiss AJ, Elixhauser A. Overview of hospital stays in the United States, 2012. HCUP statistical brief \#180. https://www.hcup-us.ahrq.gov/reports/statbriefs/ sb180-Hospitalizations-United-States-2012.jsp. Accessed April 13, 2017.

19. Hauck $K$, Zhao X. How dangerous is a day in hospital?: A model of adverse events and length of stay for medical inpatients. Med Care. 2011;49(12):1068-1075.

20. Ding A, Eisenberg JD, Pandharipande PV. The economic burden of incidentally detected findings. Radiol Clin North Am. 2011;49(2):257-265.

21. Goehler A, McMahon PM, Lumish HS, et al. Cost-effectiveness of follow-up of pulmonary nodules incidentally detected on cardiac computed tomographic angiography in patients with suspected coronary artery disease. Circulation. 2014;130(8):668-675.

22. U.S. Department of Labor. Bureau of Labor Statistics. Employment projections. Earning and unemployment rates by educational attainment, 2015. http://www.bls. gov/emp/ep_chart_001.htm. Updated March 15, 2016. Accessed April 13, 2017 